\title{
Managing blood pressure control in Asian patients: safety and efficacy of losartan
}

This article was published in the following Dove Press journal:

Clinical Interventions in Aging

19 March 2014

Number of times this article has been viewed

\author{
Tommy Tsang Cheung \\ Bernard Man Yung Cheung \\ Division of Clinical Pharmacology \\ and Therapeutics, Department of \\ Medicine, Li Ka Shing Faculty of \\ Medicine, The University of Hong \\ Kong, Hong Kong
}

\begin{abstract}
Hypertension is common in Asian populations and is a major cause of cardiovascular diseases. The prevalence of hypertension is increasing in many Asian countries. The overall prevalence of hypertension in India and the People's Republic of China has been estimated to be $20.6 \%$ in men and $22.6 \%$ in women. However, the rates of detection, treatment, and control of hypertension remain low in Asia. This reflects a low level of literacy and education, as well as a low level of access to medical care. To overcome these obstacles, strategies targeted at education, promotion, and optimization of medical care, are crucial to achieve target blood pressure control. Angiotensin receptor blockers are one of the first-line treatments for essential hypertension because they confer better cardiovascular outcomes. Losartan has been widely evaluated for the management of hypertension. Although some studies suggested that the blood pressure-lowering effect of losartan is perhaps lower than for other angiotensin receptor blockers, losartan has been demonstrated to be beneficial in terms of renal protection in patients with diabetes, heart failure resulting from either systolic or diastolic dysfunction, and diuretic-induced hyperuricemia. However, most of these data were obtained from Caucasian populations. The efficacy and safety of losartan in Asian populations may be different because of genetic and ethnic variations. Therefore, the efficacy and safety of losartan in Asian patients with hypertension warrant further study.
\end{abstract}

Keywords: hypertension, Asians, losartan

\section{Introduction}

Hypertension is common in Asian populations and is a major cause of cardiovascular diseases. The prevalence of hypertension is increasing in many countries. Nowadays, a quarter of the world's adult population has hypertension, and this percentage is likely to increase to $29 \%$ by 2025 . $^{1}$

Lifestyle modification is important for patients with hypertension. Previous studies have shown that smoking, heavy alcohol consumption, a diet high in salt, and a sedentary lifestyle are associated with hypertension. However, lifestyle modification alone is usually not sufficient to control high blood pressure. Most of the patients require antihypertensive medications to achieve the target blood pressure, and among those who require drug treatment, more than $70 \%$ of the patients require the use of two or more antihypertensive drugs. ${ }^{2}$

The British guidelines on the management of hypertension were updated in August 2011. In the new guidelines, patients younger than 55 years are recommended to receive an angiotensin-converting enzyme (ACE) inhibitor or a "low-cost" angiotensin receptor blocker as the first-line treatment. ${ }^{3}$ Blockers of the renin-angiotensin-aldosterone system (RAAS) have surpassed diuretics and beta-blockers because those agents
Yung Cheung

Division of Clinical Pharmacology and

Therapeutics, Department of Medicine,

Queen Mary Hospital, Hong Kong

Tel +85222554347

Fax +85228186474

Email mycheung@hku.hk 
were better in terms of cardiovascular outcome. Moreover, angiotensin receptor blockers (ARBs) and ACE were associated with a decrease in new-onset diabetes. ${ }^{4}$

Losartan has been widely evaluated for the treatment of hypertension. Although some studies have suggested that the blood pressure-lowering effect of losartan is perhaps lower than for other ARBs, losartan was demonstrated to be beneficial in terms of renal protection in patients with diabetes, heart failure resulting from either systolic or diastolic dysfunction, and diuretic-induced hyperuricemia. However, most of these data were obtained from Caucasian populations. The efficacy and safety of losartan in Asian populations may be different because of genetic and ethnic variations. Therefore, the efficacy and safety of losartan in Asian patients with hypertension are reviewed in this article.

\section{Prevalence of hypertension in Asia}

Hypertension is one of the major health burdens in the world. The prevalence of hypertension in developed countries is around $40 \%$, whereas its prevalence in developing countries was estimated to be $25 \% .{ }^{1}$ However, because of the larger population in developing countries, the absolute numbers of patients affected by hypertension are considerably higher, and the prevalence is likely to rise as a result of urbanization and improved life expectancy.

The prevalence of hypertension in India and the People's Republic of China has been analyzed. The overall Indian and Chinese population prevalence for hypertension was $20.6 \%$ in men and $22.6 \%$ in women. ${ }^{1}$ The prevalence of hypertension in the People's Republic of China has increased significantly in the last few years. In 1998, 24\% of the population in the People's Republic of China aged 35 to 59 years had hypertension, which was $2.3 \%$ higher than that observed from 1992 to 1994. ${ }^{5}$ In a recent analysis of the Indian cohorts, the prevalence of hypertension and prehypertension was $42.5 \%$ and $41.5 \%$, respectively. A national survey in Pakistan also showed that nowadays, $20.7 \%$ of the adult population has hypertension. ${ }^{6}$

\section{Challenges in managing hypertension in Asia}

In Asia, central or abdominal obesity is an important cause of hypertension and diabetes. ${ }^{7}$ In the Hong Kong Cardiovascular Risk Factor Prevalence Study, 58\% of people with diabetes had raised blood pressure, whereas $56 \%$ of people with hypertension had dysglycemia. ${ }^{8}$ Several reports have suggested that Chinese people and South Asians had a higher prevalence of dyslipidemia, diabetes, and hypertension at a much lower body mass index than Europeans. Therefore, the International Diabetes Federation consensus group provided a new definition of obesity for Asians with a cutoff much lower for the Chinese, Japanese, and South Asian populations compared with that for Europeans. ${ }^{9}$

As obesity is a major cause of hypertension, and as its prevalence is increasing in Asia, treatment targeting obesity will help lower the incidence of hypertension in the future. People in the community must embrace a healthy lifestyle and change their diet and leisure activities to prevent the development of obesity. Asian households tend to be larger, and it is common for the extended family to live together. This means that lifestyle changes should be targeted at the household, rather than the individual hypertensive patient. A family approach should work well in Asia.

Apart from lifestyle modifications to prevent obesity, much effort has gone into promoting the awareness of hypertension. However, the rates of detection, treatment, and control remain low in Asia. This reflected a low level of literacy and education but also reflected a low level of access to medical care. In South Asian countries, low awareness correlated with poor access to treatment. ${ }^{10}$ The challenge posed by low levels of awareness must be overcome through strategies targeted at education and promotion. Blood pressure measurement should be advocated in every adult. Because of the large population in Asian countries, ambulatory blood pressure is not a feasible option for detection and monitoring of blood pressure. Instead, home blood pressure monitoring has proved to be very useful and is increasingly affordable.

Regarding drug treatment for hypertension, Asians appear to respond better to calcium channel blockers (CCBs) but have a higher incidence of dry cough with ACE inhibitors. ${ }^{11}$ ARBs may therefore be more suitable for Asian patients.

\section{Treatment recommendation for hypertension}

The British guidelines on the management of hypertension, developed by the National Institute for Clinical Excellence, were updated August 2011. ${ }^{3}$ They are highly evidence-based and incorporate cost-effectiveness considerations. Compared with many other consensus guidelines, the British guidelines are less dependent on expert opinion but include a diverse range of opinions, including nonspecialists, nonclinicians, and the pharmaceutical industry.

In the new guidelines, there were changes to the recommended first-line treatment. As before, patients younger than 55 years are recommended to receive an ACE inhibitor or a "low-cost" ARB as the first-line treatment.

$\mathrm{CCB}$ and RAAS blockers have surpassed diuretics and beta-blockers as first-line drugs largely as a result of the 
Anglo-Scandinavian Cardiac Outcomes Trial (ASCOT), which showed that those newer agents were better than the older agents in terms of cardiovascular outcome. ${ }^{12}$ Moreover, in ASCOT and in meta-analysis, ARB and ACEI were associated with a decrease in new-onset diabetes, whereas diuretics and beta-blockers were associated with an increase in risk.

\section{Clinical efficacy of losartan in Caucasian populations Blood pressure control}

The ARBs have an effect similar to other antihypertensive monotherapies. However, several studies have shown that losartan is less effective for controlling blood pressure when compared with other ARBs such as irbesartan, telmisartan, and valsartan.

Patients receiving 8-week treatment with $300 \mathrm{mg}$ irbesartan had greater reductions in systolic and diastolic blood pressure than those receiving $100 \mathrm{mg}$ losartan, although the antihypertensive effect of $150 \mathrm{mg}$ irbesartan did not differ significantly from that of $100 \mathrm{mg}$ losartan. ${ }^{13}$

In a randomized controlled trial comparing the efficacy of telmisartan and losartan, the reductions in ambulatory systolic and diastolic blood pressure with telmisartan $40 \mathrm{mg}$ and $80 \mathrm{mg}$ were both significantly greater than those observed for losartan $50 \mathrm{mg}$. Losartan was even shown to be no better than placebo in this study. ${ }^{14}$

Another, similar, randomized controlled trial was published in 1999, comparing the efficacy of valsartan and losartan in patients with uncomplicated essential hypertension. After 4 weeks of active treatment, the medication doses were doubled. Valsartan produced a significantly higher number of responders than losartan (62\% versus [vs] 55\%; $P=0.02)$ at the 8-week treatment endpoint. Valsartan 80/160 mg monotherapies were shown to be effective and well-tolerated in management of mild to moderate essential hypertension. However, valsartan $160 \mathrm{mg}$ had a significantly higher responder rate than $100 \mathrm{mg}$ losartan. ${ }^{15}$

\section{Renal protection}

In addition to strict glycemic and blood pressure control, numerous studies have confirmed the role of renal protection by RAAS blockade. Studies in diabetic animals suggested that intraglomerular hypertension and glomerular hypertrophy play an important role in diabetic nephropathy. Therefore, reducing the intraglomerular pressure by RAAS blockade should minimize the progression of or even prevent glomerular disease in the absence of glycemic control.
Experimental studies suggest that the nondihydropyridine CCBs can also retard the progression of diabetic nephropathy. However, diltiazem alone was associated with increased tubulo-interstitial fibrosis and global glomerulosclerosis, but this effect can be prevented by combination therapy with RAAS blockers.

In the Reduction of Endpoints in Non-Insulin-Dependent Diabetes with the Angiotensin II Antagonist Losartan (RENAAL) trial, 1,513 patients with diabetic nephropathy were randomly assigned to receive either losartan or placebo in addition to conventional antihypertensive therapy. Losartan retarded the progression to end-stage renal failure by $28 \%$ and reduced the doubling of the plasma creatinine by $25 \%$ after a mean follow-up of 3.4 years. These benefits were again not associated with the differences in blood pressure levels between two treatment groups. ${ }^{16}$

\section{Treatment of heart failure resulting from systolic dysfunction}

Blockade of the RAAS system is a key component of treatment for systolic heart failure, as it improves morbidity and mortality in these patients. ARBs act through a different mechanism than ACE inhibitors, although both reduce the stimulation of angiotensin II receptors.

A meta-analysis including 1,896 patients was performed on the basis of six controlled, double-blind, multiple-dose studies, regardless of sample size or duration of follow-up. There were 36 deaths in the losartan groups compared with 47 in the control groups $(3.12 \%$ vs $6.35 \% ; P=0.004)$ during the double-blind periods. In this analysis, treatment with losartan provided a beneficial effect on survival. However, because the number of deaths in these studies was relatively small and the follow-up was relatively short, the mortality benefit of losartan remained controversial. ${ }^{17}$

In the Heart Failure Endpoint Evaluation of Angiotensin II Antagonists Losartan trial (HEAAL), slightly but significantly better outcomes were obtained with higher doses of losartan in 3,846 patients intolerant to ACE inhibitors. At a median follow-up of 4.7 years, patients receiving losartan $150 \mathrm{mg}$ had a significantly lower incidence of cardiovascular death ( $43 \%$ vs $46 \%)$ or hospitalization for heart failure (23\% vs $26 \%$ ) than those receiving losartan $50 \mathrm{mg}$. Renal dysfunction, hypotension, and hyperkalemia were more common in the $150 \mathrm{mg}$ than in the $50 \mathrm{mg}$ group, but the discontinuation rates were similar in both groups. ${ }^{18}$

In the 2012 Cochrane review, ARBs had a borderline statistically significant effect on total mortality compared with placebo, with a relative risk of 0.87 . In addition, drug 
withdrawals resulting from adverse effects were significantly less common with ARBs when compared with ACE inhibitors, with a relative risk of $0.63 .{ }^{19}$

The American College of Cardiology/American Heart Association and European Society of Cardiology therefore supported the use of ARBs in patients with current or prior symptoms of heart failure resulting from systolic dysfunction, especially in those who are intolerant of ACE inhibitors. ${ }^{20,21}$

\section{Treatment of heart failure resulting from diastolic dysfunction}

Regression of left ventricular hypertrophy is an important therapeutic target for patients with hypertension because it may improve diastolic function. A meta-analysis published in 2003 showed that angiotensin II receptor blockers were the most effective drug class for the regression of left ventricular hypertrophy, followed by calcium channel blockers and ACE inhibitors. ${ }^{22}$

Losartan has been extensively evaluated for its benefit in heart failure resulting from diastolic dysfunction. After 1 year of losartan-based regimen for hypertension, parameters of diastolic function as measured by echocardiogram improved in patients with reductions in left ventricular mass. ${ }^{23}$ This finding was supported by another study, which evaluated the myocardial stiffness quantified by transvenous endomyocardial biopsy. After 1-year treatment with losartan, collagen volume fraction and left ventricular stiffness significantly decreased in patients with severe myocardial fibrosis. Therefore, losartan has the ability to induce regression of severe myocardial fibrosis, resulting in diminution of myocardial stiffness in hypertensive patients. ${ }^{24}$

The clinical benefit of losartan was demonstrated by a large randomized controlled trial aiming to compare the efficacy of a losartan-based regimen with that of an atenololbased regimen. Losartan was shown to prevent more cardiovascular morbidity and death than atenolol for a similar reduction in blood pressure, and it was better tolerated. ${ }^{25} \mathrm{In}$ addition, improved quality of life in patients with heart failure resulting from diastolic dysfunction was also demonstrated in small, randomized control trials. ${ }^{26,27}$

\section{Urate-lowering effect of losartan}

Among patients with hypertension, the concurrent use of losartan can reduce plasma urate levels; the use of other ARBs or ACE inhibitors does not. In a small prospective study comparing the urate-lowering effect of losartan and irbesartan, 4 weeks of losartan decreased serum uric acid levels by $9 \%$, whereas irbesartan had no effect. ${ }^{28}$ In an in vitro study, losartan, but not other ARBs, inhibited the renal urate anion exchanger that partially mediates urate reabsorption. ${ }^{29}$

In a randomized controlled trial comparing the efficacy of enalapril and losartan in patients with hypertension, losartan, but not enalapril, reduced plasma urate levels; there was no significant difference in other metabolic parameters between the two groups..$^{30}$

The urate-lowering effect of losartan was also demonstrated in patients receiving diuretics. One thousand one hundred and sixty-one patients with mild to moderate hypertension were randomly assigned to receive losartan, losartan plus hydrochlorothiazide, or candesartan for 12 weeks. The serum urate levels decreased, remained the same, or increased in those given losartan, losartan plus hydrochlorothiazide, and candesartan, respectively. ${ }^{31}$ Although uric acid excretion is initially increased, there appears to be little risk for uric acid nephropathy with losartan because of the concurrent elevation in urine $\mathrm{pH}$ resulting from reduced bicarbonate reabsorption. ${ }^{32}$ As a result, there was little or no increase in the urinary excretion of insoluble uric acid.

\section{Safety and tolerability of losartan}

The incidence of cough is lower in patients treated with ARBs. Although there was no direct comparison between losartan and other ACE inhibitors, the magnitude of this difference was demonstrated in a meta-analysis that directly compared the rate of cough in ARBs and ACE inhibitors. ${ }^{33}$ The rates of cough were $3.2 \%$ and $9.9 \%$, respectively, although there was significant statistical heterogeneity among the studies included in the meta-analysis.

The risk for angioedema appears to be lower with ARBs than ACE inhibitors. However, angioedema had been reported with losartan. ${ }^{34}$ The pathogenesis of angioedema with ARBs is unclear, but an increase in tissue bradykinin levels or nonkinin factors may be involved.

Hypotension appears to be more common with ARBs than ACE inhibitors. However, most of the patients who developed hypotension were taking multiple antihypertensive drugs and had other comorbidities. Therefore, there was no conclusive evidence to support the idea that the risk for hypotension is more common with ARBs.

\section{Clinical efficacy of losartan in Asian populations Blood pressure control}

Although there was no direct comparison of losartan with other ARBs for the treatment of hypertension in an Asian population, a multicenter study recruiting more than 200 Asian patients in the People's Republic of China, South 
Korea, Taiwan, and Peru showed that losartan $50 \mathrm{mg}$ plus hydrochlorothiazide $12.5 \mathrm{mg}$ was superior to valsartan $80 \mathrm{mg}$ in the treatment of hypertension.

Patients with suboptimal control of blood pressure with valsartan $80 \mathrm{mg}$ were switched to a losartan plus hydrochlorothiazide combination. The mean reductions after switching were 8.1 and $8.3 \mathrm{mmHg}$ for systolic and diastolic blood pressure, respectively. The goal of diastolic blood pressure was achieved in 72 of the patients with hypertension previously not controlled by valsartan $80 \mathrm{mg}$. Combination therapy was generally welltolerated, with a mean compliance rate of more than $99 \%{ }^{35}$

Otherwise, most of the studies on the efficacy and safety of losartan were conducted in the Japanese cohorts. They demonstrated similar clinical efficacy of losartan, as mentioned earlier.

Another, similar, study was published in 2009, comparing the efficacy of the losartan plus hydrochlorothiazide combination with other ACE inhibitors and ARBs. In this 12-week, open-label trial, patients with suboptimal control of blood pressure despite the use of ACE inhibitors or ARB monotherapy were switched to losartan $50 \mathrm{mg}$ plus hydrochlorothiazide $12.5 \mathrm{mg}$. At 4 and 8 weeks, patients who did not achieve target blood pressure were given losartan $100 \mathrm{mg}$ plus hydrochlorothiazide $12.5 \mathrm{mg}$ and losartan $100 \mathrm{mg}$ plus hydrochlorothiazide $25 \mathrm{mg}$, respectively. After 8 weeks, $73.5 \%$ of the patients reached their blood pressure goal; $78.1 \%$ of the patients reached their blood pressure goal at 12 weeks. The mean reductions from baseline systolic and diastolic blood pressure at 8 weeks were 16.7 and $12.1 \mathrm{mmHg}$, respectively.

This study suggested that switching Asian patients with suboptimal blood control with ACE inhibitors or ARBs to a losartan plus hydrochlorothiazide combination was more effective without compromising tolerability.

\section{Renal protection}

A study conducted in Singapore compared the renal protective efficacy of losartan with quinapril. Forty-one ARB- and ACE inhibitor-naïve participants with type 2 diabetes and albuminuria were given either $50 \mathrm{mg}$ losartan or $20 \mathrm{mg}$ quinapril for 4 weeks, with a 4 -week wash-out period between interventions in a crossover fashion.

The use of losartan showed a significant reduction in albuminuria, as measured by urine protein over creatinine ratio, when compared with the use of quinapril $(-93 \mathrm{mg} / \mathrm{g}$ vs $-49 \mathrm{mg} / \mathrm{g} ; P=0.025)$. The reduction in albuminuria was not associated with the reduction in blood pressure levels, as the systolic and diastolic blood pressure reductions were similar between two treatment groups. However, because of the short study duration, the long-term efficacy of losartan in renal protection should be further evaluated. ${ }^{36}$

The RENAAL study was a double-blind, randomized, placebo-controlled trial comparing the renal protective effects of losartan on a background of conventional antihypertensive therapies in more than 1,500 patients with type 2 diabetes and nephropathy. In this multiethnic trial, 252 Asian participants were recruited, most of whom were from Japan, Hong Kong, Malaysia, and Singapore. ${ }^{37}$

Although there was no significant difference in blood pressure reduction and the composite endpoints of cardiovascular mortality and morbidity between two groups, losartan reduced the risk of doubling of serum creatinine, end-stage renal disease, or all-cause mortality in Asian patients by $35 \%$ ( $P=0.02)$. The use of losartan also reduced the level of proteinuria by $47 \%(P<0.001)$ and the rate of decrease in renal function by $31 \%(P=0.007) .{ }^{37}$

Another study had evaluated the efficacy of losartan plus conventional antihypertensive therapies in the cost reduction associated with end-stage renal disease. The use of losartan reduced the number of days with end-stage renal failure by 37.9 per patient over the course of 3.5 years compared with conventional antihypertensive therapies. This reduction resulted in a decrease in the cost ranging from $\$ 910$ to $\$ 4,346$ per patient over the six regions. After calculation of the cost of losartan, the net savings in each of the six regions ranged from $\$ 55$ to $\$ 515$ per patient. ${ }^{38}$

The effects of losartan plus hydrochlorothiazide were compared with losartan alone in Japanese diabetic patients with hypertension. Thirty Japanese diabetic patients with hypertension were randomly assigned to receive losartan alone for the first 3 months and then combination therapy for the next 3 months, or vice versa. The decreases in systolic and diastolic blood pressure during combination treatment were significantly greater than in treatment with losartan alone. However, both treatment groups had significant and similar decrease in urinary albumin excretion, although there was no significant difference in metabolic change during the monoand combination therapies. ${ }^{39}$ This study also confirmed that the efficacy of losartan in renal protection is independent of the reductions in blood pressure levels.

\section{Treatment of heart failure resulting from diastolic dysfunction}

Losartan was compared with amlodipine in a prospective, randomized study to assess the effects on left ventricular diastolic function and atherosclerosis of the carotid artery in patients with mild to moderate hypertension. A total 
of 57 patients were randomly assigned to losartan- or amlodipine-based treatment groups and were followed-up for 18 months. Blood pressure was similarly reduced by both regimens. Although losartan shortened the transmitral E-wave deceleration time and amlodipine reduced left ventricular mass index, there was no significant difference in these parameters between the two groups. However, the mean carotid intima-media thickness as well as plaque score significantly increased in the amlodipine-based treatment groups, but not in the losartan-based treatment groups. Therefore, under similar reduction of blood pressure, losartan is likely effective in protecting the progression of atherosclerosis of the carotid artery compared with amlodipine, but the exact mechanism of this observation has not been fully explained by this study. ${ }^{40}$

\section{Urate-lowering effect of losartan}

A multicenter, randomized, double-blind controlled trial was conducted in the People's Republic of China to compare the urate-lowering effects of losartan and irbesartan in Chinese patients with mild to moderate essential hypertension. In this study, 162 Chinese patients were randomly assigned to the losartan group, and 163 patients were randomly assigned to the irbesartan group.

This study showed that losartan $50 \mathrm{mg}$ once daily produced a significant decrease in serum urate levels from $422 \mathrm{mmol} / \mathrm{L}$ at baseline to $367 \mathrm{mmol} / \mathrm{L}$ at week 4 ( $P=0.0001)$. The use of losartan 50 or $100 \mathrm{mg}$ once daily for 8 weeks further decreased the serum urate levels to $359 \mathrm{mmol} / \mathrm{L}$. However, there was no significant change in serum urate levels in the irbesartan group throughout the study. ${ }^{41}$

\section{Safety and tolerability of losartan in Asian populations}

Data from the RENAAL study showed that Asian patients had the lowest rates of discontinuation. In Asian patients, losartan was well-tolerated with no increase in incidence of cough. ${ }^{37}$ In India, losartan was proven to be effective and safe. Data were obtained in 347 patients from 140 general physicians. The study revealed that losartan potassium was used in the treatment of mild to moderate hypertension with excellent to good response in $98.8 \%$ of the cases. Mild adverse reactions were reported in $5.8 \%$ of the cases. None of the adverse reactions were severe enough to require discontinuation of therapy or to need hospitalization. ${ }^{42}$

Another study from Japan evaluated the long-term safety of losartan plus hydrochlorothiazide combinations.
After 1 year of treatment, only $11 \%$ of the patients developed clinical and laboratory adverse events, but serious abnormalities were not observed. ${ }^{43}$

\section{Conclusion}

The health burden of hypertension in Asia is increasing as a result of changes in lifestyle, increased incidence of obesity, and improved life expectancy. To optimize the control of hypertension, appropriate use of antihypertensive medications is crucial. New National Institute for Clinical Excellence guidelines suggested the use of ACE inhibitors or ARBs as the first-line treatment for essential hypertension. However, a higher incidence of dry cough with ACE inhibitors was reported in Asian populations. Therefore, ARB may be more suitable for Asian patients with hypertension.

The efficacy and safety have been evaluated in several studies, mainly in Japanese cohorts. Losartan or a losartan plus hydrochlorothiazide combination are effective in Asian patients with essential hypertension. In addition, other benefits of losartan in terms of renal protection in patients with diabetes and treatment of heart failure resulting from diastolic dysfunction were also demonstrated in Asian populations. More important, this drug was proven to be safe and welltolerated in Asian patients.

\section{Disclosure}

The authors report no conflicts of interest in this work.

\section{References}

1. Kearney PM, Whelton M, Reynolds K, Muntner P, Whelton PK, He J. Global burden of hypertension: analysis of worldwide data. Lancet. 2005;365(9455):217-223.

2. Tsang Cheung T, Man Yung Cheung B. Identifying patients with resistant hypertension and options for clinical management. Future Cardiol. 2012;8(6):837-846.

3. Ritchie LD, Campbell NC, Murchie P. New NICE guidelines for hypertension. BMJ. 2011;343:d5644.

4. McCormack T, Krause T, O'Flynn N. Management of hypertension in adults in primary care: NICE guideline. Br J Gen Pract. 2012;62(596): 163-164

5. Wang Z, Wu Y, Zhao L, Li Y, Yang J, Zhou B; Cooperative Research Group of the Study on Trends of Cardiovascular Diseases in China and Preventive Strategy for the 21st Century. Trends in prevalence, awareness, treatment and control of hypertension in the middle-aged population of China, 1992-1998. Hypertens Res. 2004;27(10):703-709.

6. Ahmad K, Jafar TH. Prevalence and determinants of blood pressure screening in Pakistan. J Hypertens. 2005;23(11):1979-1984.

7. Cheung BM, Wat NM, Man YB, et al. Relationship between the metabolic syndrome and the development of hypertension in the Hong Kong Cardiovascular Risk Factor Prevalence Study-2 (CRISPS2). Am J Hypertens. 2008;21(1):17-22.

8. Cheung BM, Wat NM, Tso AW, et al. Association between raised blood pressure and dysglycemia in Hong Kong Chinese. Diabetes Care. 2008;31(9):1889-1891. 
9. Alberti KG, Zimmet P, Shaw J; IDF Epidemiology Task Force Consensus Group. The metabolic syndrome - a new worldwide definition. Lancet. 2005;366(9491):1059-1062.

10. Sun Z, Zheng L, Wei Y, et al. The prevalence of prehypertension and hypertension among rural adults in Liaoning province of China. Clin Cardiol. 2007;30(4):183-187.

11. Woo KS, Nicholls MG. High prevalence of persistent cough with angiotensin converting enzyme inhibitors in Chinese. Br J Clin Pharmacol. 1995;40(2):141-144.

12. Dahlöf B, Sever PS, Poulter NR, et al; ASCOT Investigators. Prevention of cardiovascular events with an antihypertensive regimen of amlodipine adding perindopril as required versus atenolol adding bendroflumethiazide as required, in the Anglo-Scandinavian Cardiac Outcomes Trial-Blood Pressure Lowering Arm (ASCOT-BPLA) a multicentre randomised controlled trial. Lancet. 2005;366(9489): 895-906.

13. Kassler-Taub K, Littlejohn T, Elliott W, Ruddy T, Adler E. Comparative efficacy of two angiotensin II receptor antagonists, irbesartan and losartan in mild-to-moderate hypertension. Irbesartan/Losartan Study Investigators. Am J Hypertens. 1998;11(4 Pt 1):445-453.

14. Mallion J, Siche J, Lacourcière Y. ABPM comparison of the antihypertensive profiles of the selective angiotensin II receptor antagonists telmisartan and losartan in patients with mild-to-moderate hypertension. J Hum Hypertens. 1999;13(10):657-664.

15. Hedner T, Oparil S, Rasmussen K, et al. A comparison of the angiotensin II antagonists valsartan and losartan in the treatment of essential hypertension. Am J Hypertens. 1999;12(4 Pt 1):414-417.

16. Brenner BM, Cooper ME, de Zeeuw D, et al; RENAAL Study Investigators. Effects of losartan on renal and cardiovascular outcomes in patients with type 2 diabetes and nephropathy. $N$ Engl $J$ Med. 2001;345(12):861-869.

17. Sharma D, Buyse M, Pitt B, Rucinska EJ. Meta-analysis of observed mortality data from all-controlled, double-blind, multiple-dose studies of losartan in heart failure. Losartan Heart Failure Mortality Meta-analysis Study Group. Am J Cardiol. 2000;85(2):187-192.

18. Konstam MA, Neaton JD, Dickstein K, et al; HEAAL Investigators. Effects of high-dose versus low-dose losartan on clinical outcomes in patients with heart failure (HEAAL study): a randomised, double-blind trial. Lancet. 2009;374(9704):1840-1848.

19. Heran BS, Musini VM, Bassett K, Taylor RS, Wright JM. Angiotensin receptor blockers for heart failure. Cochrane Database Syst Rev. 2012;4:CD003040

20. Hunt SA, Abraham WT, Chin MH, et al; American College of Cardiology Foundation; American Heart Association. 2009 Focused update incorporated into the ACC/AHA 2005 Guidelines for the Diagnosis and Management of Heart Failure in Adults A Report of the American College of Cardiology Foundation/American Heart Association Task Force on Practice Guidelines Developed in Collaboration With the International Society for Heart and Lung Transplantation. $J$ Am Coll Cardiol. 2009;53(15):e1-e90.

21. McMurray JJ, Adamopoulos S, Anker SD, et al; Task Force for the Diagnosis and Treatment of Acute and Chronic Heart Failure 2012 of the European Society of Cardiology; ESC Committee for Practice Guidelines. ESC guidelines for the diagnosis and treatment of acute and chronic heart failure 2012: The Task Force for the Diagnosis and Treatment of Acute and Chronic Heart Failure 2012 of the European Society of Cardiology. Developed in collaboration with the Heart Failure Association (HFA) of the ESC. Eur J Heart Fail. 2012;14(8): 803-869.

22. Klingbeil AU, Schneider M, Martus P, Messerli FH, Schmieder RE. A meta-analysis of the effects of treatment on left ventricular mass in essential hypertension. Am J Med. 2003;115(1):41-46.

23. Wachtell K, Bella JN, Rokkedal J, et al. Change in diastolic left ventricular filling after one year of antihypertensive treatment: The Losartan Intervention For Endpoint Reduction in Hypertension (LIFE) Study. Circulation. 2002;105(9):1071-1076.
24. Díez J, Querejeta R, López B, et al. Losartan-dependent regression of myocardial fibrosis is associated with reduction of left ventricular chamber stiffness in hypertensive patients. Circulation. 2002;105(21):2512-2517.

25. Dahlöf B, Devereux RB, Kjeldsen SE, et al; LIFE Study Group. Cardiovascular morbidity and mortality in the Losartan Intervention For Endpoint reduction in hypertension study (LIFE): a randomised trial against atenolol. Lancet. 2002;359(9311):995-1003.

26. Warner JG Jr, Metzger DC, Kitzman DW, Wesley DJ, Little WC. Losartan improves exercise tolerance in patients with diastolic dysfunction and a hypertensive response to exercise. J Am Coll Cardiol. 1999;33(6):1567-1572.

27. Little WC, Zile MR, Klein A, Appleton CP, Kitzman DW, WesleyFarrington DJ. Effect of losartan and hydrochlorothiazide on exercise tolerance in exertional hypertension and left ventricular diastolic dysfunction. Am J Cardiol. 2006;98(3):383-385.

28. Würzner G, Gerster JC, Chiolero A, et al. Comparative effects of losartan and irbesartan on serum uric acid in hypertensive patients with hyperuricaemia and gout. J Hypertens. 2001;19(10):1855-1860.

29. Enomoto A, Kimura H, Chairoungdua A, et al. Molecular identification of a renal urate anion exchanger that regulates blood urate levels. Nature. 2002;417(6887):447-452.

30. Tikkanen I, Omvik P, Jensen HA. Comparison of the angiotensin II antagonist losartan with the angiotensin converting enzyme inhibitor enalapril in patients with essential hypertension. J Hypertens. 1995; 13(11):1343-1351.

31. Manolis AJ, Grossman E, Jelakovic B, et al. Effects of losartan and candesartan monotherapy and losartan/hydrochlorothiazide combination therapy in patients with mild to moderate hypertension. Losartan Trial Investigators. Clin Ther. 2000;22(10):1186-1203.

32. Shahinfar S, Simpson RL, Carides AD, et al. Safety of losartan in hypertensive patients with thiazide-induced hyperuricemia. Kidney Int. 1999;56(5):1879-1885.

33. Matchar DB, McCrory DC, Orlando LA, et al. Systematic review: comparative effectiveness of angiotensin-converting enzyme inhibitors and angiotensin II receptor blockers for treating essential hypertension. Ann Intern Med. 2008;148(1):16-29.

34. van Rijnsoever EW, Kwee-Zuiderwijk WJ, Feenstra J. Angioneurotic edema attributed to the use of losartan. Arch Intern Med. 1998;158(18): 2063-2065.

35. Watanabe LA, Wei M, Sun N, et al. Effect on blood pressure control of switching from valsartan monotherapy to losartan/hydrochlorothiazide in Asian patients with hypertension: results of a multicentre open-label trial. Curr Med Res Opin. 2006;22(10):1955-1964.

36. Lim SC, Koh AF, Goh SK, et al. Angiotensin receptor antagonist vs angiotensin-converting enzyme inhibitor in Asian subjects with type 2 diabetes and albuminuria - a randomized crossover study. Diabetes Obes Metab. 2007;9(4):477-482.

37. Chan JC, Wat NM, So WY, et al; Asian RENAAL Study Investigators. Renin angiotensin aldosterone system blockade and renal disease in patients with type 2 diabetes. An Asian perspective from the RENAAL Study. Diabetes Care. 2004;27(4):874-879.

38. Seng WK, Hwang SJ, Han DC, et al. Losartan reduces the costs of diabetic end-stage renal disease: an Asian perspective. Nephrology (Carlton). 2005;10(5):520-524.

39. Kinouchi K, Ichihara A, Sakoda M, Kurauchi-Mito A, Itoh H. Safety and benefits of a tablet combining losartan and hydrochlorothiazide in Japanese diabetic patients with hypertension. Hypertens Res. 2009; 32(12):1143-1147.

40. Yamamoto K, Ozaki H, Takayasu K, et al. The effect of losartan and amlodipine on left ventricular diastolic function and atherosclerosis in Japanese patients with mild-to-moderate hypertension (J-ELAN) study. Hypertens Res. 2011;34(3):325-330.

41. Dang A, Zhang Y, Liu G, Chen G, Song W, Wang B. Effects of losartan and irbesartan on serum uric acid in hypertensive patients with hyperuricaemia in Chinese population. J Hum Hypertens. 2006;20(1): $45-50$. 
42. Joshi SR, Kale S, Oomman A, Joshi RM, Chopra D. Study of efficacy and safety of losartan (losar) in Asian Indian hypertensives. J Indian Med Assoc. 2005;103(7):392, 394-396.

43. Kita T, Yokota N, Ichiki Y, et al. One-year effectiveness and safety of open-label losartan/hydrochlorothiazide combination therapy in Japanese patients with hypertension uncontrolled with ARBs or ACE inhibitors. Hypertens Res. 2010;33(4):320-325.

\section{Publish your work in this journal}

Clinical Interventions in Aging is an international, peer-reviewed journal focusing on evidence-based reports on the value or lack thereof of treatments intended to prevent or delay the onset of maladaptive correlates of aging in human beings. This journal is indexed on PubMed Central, MedLine, the American Chemical Society's 'Chemical Abstracts Ser-

vice' (CAS), Scopus and the Elsevier Bibliographic databases. The manuscript management system is completely online and includes a very quick and fair peer-review system, which is all easy to use. Visit http://www.dovepress.com/testimonials.php to read real quotes from published authors.

Submit your manuscript here: http://www.dovepress.com/clinical-interventions-in-aging-journal 